\title{
Lorazepam Strongly Prolongs Visual Information Processing
}

\author{
Anne Giersch*,' and Michael H Herzog ${ }^{2}$ \\ 'Department of Psychiatry, Hôpitaux Universitaires de Strasbourg, Strasbourg Cedex, France; ' Human Neurobiology, University of Bremen, \\ Bremen, Germany
}

\begin{abstract}
Lorazepam is a drug that has been widely used for over 30 years. Whereas its therapeutic and amnestic effects are fairly well known, the visuo-perceptual impairments induced by this drug have been studied to a much lesser degree and only little is known about the influence of lorazepam on the time course of visual information processing. To gain a better insight specifically on these temporal characteristics, we used the recently discovered backward masking technique, 'shine-through', in which a vernier target precedes a grating. We tested subjects, treated with lorazepam, diazepam, or a placebo, with masking gratings of various spatial layouts. Our experiments reveal surprising results. First, for the unmasked vernier target, lorazepam induced a strong deterioration of performance compared to both diazepam and placebo. Performance deteriorated even more significantly if a masking grating was presented following the vernier. We observed that vernier discrimination could be completely abolished even if the grating appeared $400 \mathrm{~ms}$ after the vernier presentation. Such long time intervals are beyond usual visual masking effects. When performing the task under placebo, the participants perceived the vernier target and the masking grating as two independent time events rather than as a single event. It appears that lorazepam prolongs dramatically the processing of visual targets. The masking effects revealed here are specific to the type of grating and are much stronger under lorazepam than under diazepam.

Neuropsychopharmacology (2004) 29, I 386-1394, advance online publication, 3 I March 2004; doi: I 0. I 038/sj.npp. I 300429
\end{abstract}

Keywords: lorazepam; diazepam; benzodiazepine; backward masking; visual perception; shine-through effect

\section{INTRODUCTION}

Lorazepam and diazepam are drugs that are characterized, as are all benzodiazepines, by hypnotic, anxiolytic, myorelaxant, and antiepileptic properties. Both drugs act through the enhancement of GABA fixation, the main inhibitory neurotransmittor of the $\mathrm{GABA}_{\mathrm{A}}$ receptor. Lorazepam and diazepam have no intrinsic properties when taken at therapeutic doses and their acute effect is totally reversible.

Whereas the effects of lorazepam on spatial information perception have been investigated for over 10 years, the effects of this benzodiazepine on the time course for the processing of visual information have only rarely been investigated. Fisch et al (1983) and Emre et al (1989) described increased backward masking after the intake of both diazepam and midazolam. Treated subjects needed more time between the presentation of target and mask (ie longer interstimulus interval, ISI) in order to identify the target (Emre et al, 1989). Subjects treated with benzodia-

\footnotetext{
* Correspondence: A Giersch, Département de Psychiatrie I, Hôpitaux Universitaires de Strasbourg, INSERM U405, I, pl de l'Hôpital, 6709 | Strasbourg Cedex, France, Tel.: + 33388 II 647 1, Fax: + 33388 II 64 46, E-mail: giersch@alsace.u-strasbg.fr

Received 08 September 2003; revised 13 January 2004; accepted 30 January 2004

Online publication: 6 February 2004 at http://www.acnp.org/citations/ Npp020604034I0/default.pdf
}

zepines have also been shown to need more time to distinguish between two consecutive events, that is, treated participants have lower fusion frequency (Danjou et al, 1992; Berlin et al, 1993; Maddock et al, 1993; Curran et al, 1993, 1998; MacNab et al, 1985). These two effects suggest that benzodiazepines may affect the time course for the processing of visual information. However, the precise temporal characteristics of the impairments induced by these benzodiazepines are only poorly understood. It might be that benzodiazepines simply slow information processing. In addition, deteriorated performance may be related to impaired decision rather than information processes per se. In this case, the effects could be regarded as nonspecific. On the other hand, lorazepam may influence information processing specifically, for example, the drug could affect specific spatial information processes such as perceptual grouping or figure-ground segmentation. Indeed, it has been shown that lorazepam impairs visuo-perceptual integration processes (Giersch et al, 1997; Giersch and Lorenceau, 1999; Beckers et al, 2001; Giersch, 1999, 2001).

In order to study the temporal aspects of the effects induced by benzodiazepines, we used a backward masking technique. In backward masking, the mask can influence target processing in different ways. Deteriorated performance can occur because the mask and the target appear as superimposed and, hence, the visual system cannot separate one from the other. In such a case, the target is harder to detect since it is, for example, camouflaged by the mask or 
the contrast of the target decreases due to the additional luminance of the mask. This type of masking is especially powerful when the mask has a higher energy level than the target, and it may be caused by the long-lasting responses of the photoreceptors. When talking about the energy of an element, we refer to the product of the luminance times the duration of presentation of this element. The energy of the mask is the total sum of the energy of each of its elements. In this rather nonspecific masking paradigm, performance improves for longer stimulus-onset asynchronies (SOA), that is, the mask is presented later relative to the target onset (eg Breitmeyer, 1984; Bachmann, 1994). A mask can also have a more specific effect on target processing, for example, it can affect the processing of its contours or even erase the representation of the target in working memory (eg Enns and Di Lollo, 2000; Breitmeyer and Ogmen, 2000; Herzog et al, 2001). Masks that are related to the processing of contours often have an energy level comparable or weaker to that of the target (eg Francis, 2000). In this type of masking, worst performances often occur when the mask follows the target by 30-80 ms.

With the aim to study the specific effects of a mask on a target under the intake of benzodiazepines, it was necessary to adopt a protocol that would enable the dissociation of the nonspecific from the specific effects of both the drugs and the masks. The recently discovered shine-through effect provided this. Indeed, in this paradigm, the spatial layout of the mask determines performance, as the energy of the mask per se does not play a role (Herzog and Koch, 2001; Francis and Herzog, in press).

In the shine-through effect, a vernier-target, that is, two abutting lines, precedes a grating comprised of aligned verniers. Surprisingly, performance is by far better if the grating contains 25 elements than if the grating is comprised of only five elements - even though the masking energy of a 25-element grating is significantly higher than for a five-element grating (Figure 1; Herzog and Koch, 2001; for an animation of the stimuli see http://neuro.physik.unibremen.de/ vernier/vernier_english/vindex.html). However, the presence of small deviations within the homogeneity of the 25-element grating, for example, the insertion of gaps within the grating, significantly deteriorates the shinethrough effect (Figure 1). Even if the energy of the grating with gaps is identical to that without gaps, performance levels are significantly deteriorated. Herzog et al (2001) suggested that the spatial layout, namely the contours and edges of the grating, determine the visibility of the shinethrough element. The key idea is that the neural activity corresponding to the processing of object contours is dynamically enhanced whereas the activity of inner grating a

\begin{tabular}{|c|c|c|c|}
\hline $0-30 \mathrm{~ms}$ & 1 & I & 1 \\
\hline $30-330 \mathrm{~ms}$ & 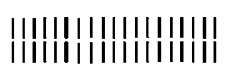 & IIIII & ling l|lil Imin \\
\hline
\end{tabular}

Figure I The shine-through effect: a vernier is followed by a grating comprised of 25 elements (a). The vernier shines through the grating appearing as wider and brighter. The participants' task is to discriminate the offset direction of the shine-through element. Gratings with five (b) or 25 elements with gaps (c) yield significantly worse performances than 25element gratings without gaps (a). elements, or more generally of redundant elements, diminishes. If such enhanced activity is close to the activity corresponding to the vernier, as it is the case for small gratings or gratings with gaps, suppression occurs. This hypothesis has been verified through computer simulations (Herzog et al, 2003). Functionally speaking, edges indicate a change from, for example, figure to background. The importance of edges has also been revealed in other psychophysical and neurophysiological investigations (MacKay, 1973; McCarter and Roehrs, 1976; Sagi and Hochstein, 1985; Macknik et al, 2000). Hence, the shinethrough effect has revealed to be a powerful tool for the investigation of the time course of spatial information processing.

To investigate the effects of benzodiazepines on information processing, we used both lorazepam and diazepam, as it is well known that lorazepam only deteriorates spatial information processing. Indeed, lorazepam has been shown to impair the identification of fragmented pictures (Vidailhet et al, 1994; Wagemans et al, 1998). These observations initiated a series of experiments, which have now shown that lorazepam globally affects processing of object contours (Giersch et al, 1997; Giersch and Lorenceau, 1999; Beckers et al, 2001; Giersch, 1999, 2001) and may increase the enhancement of edges. Lorazepam facilitates the detection of discontinuities that are located between collinear elements compared to the case where these discontinuities are present between parallel elements (Giersch, 1999; Beckers et al, 2001; Giersch, 2001). As a consequence, it has been suggested that lorazepam impairs the processes that intervene in the integration of elements into a global configuration. This has been shown in a variety of experiments, for example, with compound letters in which small letters are spatially organized to form a large letter (Giersch et al, 1997). Intake of lorazepam also results in difficulties to recover global direction from local motion signals (Giersch and Lorenceau, 1999).

Several arguments have been used to eliminate alternative explanations for these results in terms of nonspecific effects of lorazepam. First, the effects of lorazepam depend on the location of line-ends but not on the size of the stimuli. This suggests that results cannot be accounted for by an effect on spatial frequency processing (Giersch et al, 1997; Giersch, 1999). In motion experiments, the recovery of global motion was preserved under lorazepam as long as line-ends were discarded from processing (Giersch and Lorenceau, 1999). These results suggested that the effect of lorazepam cannot be attributed solely to a deficit in the processing of low spatial frequency information. Second, results in participants treated with lorazepam were not a simple exacerbation of a similar trend in placebo-treated participants, as might have been the case for a nonspecific effect. For example, there was no difference in performance for gaps between collinear or parallel elements in placebo-treated participants. Third, performance in both the identification of fragmented pictures (Vidailhet et al, 1994; Legrand et al, 1995; Wagemans et al, 1998) and the detection of discontinuities (Beckers et al, 2001) differed in lorazepamand diazepam-treated participants under comparable conditions, that is, the dose of each drug was determined to yield a comparable sedation level (Vidailhet et al, 1994). If the effects were of nonspecific origin, the results should 
have been similar under lorazepam and diazepam. Consequently and as a whole, these results demonstrate a clear difference between the diazepam and the lorazepam drugs, with furthermore the indication that lorazepam affects the processing of visual contours in a specific way independently from its sedative and other possible nonspecific effects.

The rationale and the procedure of the following experiments are as follows. First, we tested the effects of lorazepam and diazepam on the unmasked vernier target. Second, we compared the masking effect of a five- and of a 25-element grating. If lorazepam affects contour processing in the shine-through effect, the decrease in the participants' performance between the 25- and the five-element grating should be more severe under lorazepam than under both diazepam and placebo. In contrast, if benzodiazepines enhance masking in a nonspecific way, both benzodiazepines (lorazepam and diazepam) should induce an equivalent or a larger decrease in performance for the highenergy 25-element grating as compared to the low-energy five-element grating. Therefore, (1) by using high- and lowenergy masks, which also have different contour characteristics and (2) by using two different drugs, of which only one affects contour processing, we will be able to discuss the differential effects of the two benzodiazepines on the time course of the processes involved in masking and contour processing.

\section{MATERIALS AND METHODS}

\section{General Setup}

Visual stimuli were displayed on a color monitor (Sony CPD-2403) controlled by a pentium 3-733 MHz microprocessor. During the experiments, a vertical vernier was presented alone or was followed by a five- or a 25-element grating (see Figure 1). In the present experiments, vertical verniers are composed of two vertical bars that are offset in the horizontal direction. The gratings were aligned verniers, that is, verniers without horizontal offset. The horizontal distance between the different elements of the gratings was $200^{\prime \prime}$ (arc sec). Vernier and grating elements were 600" long and separated by a vertical gap of $60^{\prime \prime}$. Hence, the total length of the vernier and grating elements was $1260^{\prime \prime}$. Width of the verniers was 1 pixel. The vernier and the central element of the grating appeared always in the center of the screen. The presentation of the gratings lasted $300 \mathrm{~ms}$. Participants observed the stimuli from a distance of $250 \mathrm{~cm}$, in a dark room. From this distance, a pixel was equivalent to about $24^{\prime \prime}$. Visual stimuli were white presented on a black background $\left(0.001 \mathrm{~cd} / \mathrm{m}^{2}\right)$. The luminance of these various stimuli was approximately $90 \mathrm{~cd} / \mathrm{m}^{2}$. Refreshment rate was $100 \mathrm{~Hz}$. The participants' task was to discriminate the offset direction of the lower part of the vernier in relation to the upper segment by pressing one of two push buttons. Auditory error feedback was provided.

\section{Verniers Without Mask}

In the first part of each session, the vernier duration required for each participant was determined in order for him/she to reach a preset criterion level. Thresholds for vernier offset discrimination were determined using the adaptive method PEST (Taylor and Creelman, 1967). For a constant vernier duration, PEST offered a starting vernier offset of $150^{\prime \prime}$, which was afterwards decreased or increased to find the threshold defined as the vernier offset for which $75 \%$ correct responses were reached. Using probit analysis, we fitted psychometric functions to the data, which allowed the computation of this threshold. As this method interpolates between data points, it was also possible to determine thresholds between multiples of $24^{\prime \prime}$, that is, the pixel size. For offsets in the range above $24^{\prime \prime}$, this procedure yields reliable results.

The duration of vernier presentation was fixed to $20 \mathrm{~ms}$ in the first block. For each participant, the aim was to find the vernier duration for which the threshold for vernier offset discrimination was approximately $40^{\prime \prime}$. If the preset criterion was not reached, a block was repeated using a longer vernier duration. The lower temporal limit for vernier presentation was set to $20 \mathrm{~ms}$. In order to be included in the masking experiments, a vernier duration shorter than $100 \mathrm{~ms}$ had to be reached. Thresholds were determined within a block of 80 trials.

\section{SOA Determination-Masking Experiments}

As described above, we first determined the vernier duration for each participant. In the following experiments, we masked the vernier with either the five- or the 25element grating. The vernier offset was fixed to $75^{\prime \prime}$, throughout the whole experiment and for all participants $\left(75^{\prime \prime}\right.$ is about three times the pixel size). For all participants, this offset size was found to be larger than the offset reached in the first condition during which the vernier duration was determined without mask. As we adjusted the vernier duration for each participant individually and used a large vernier offset size, discrimination of the nonmasked vernier offset was comparable among observers. This procedure gave the means to dissociate the vernier offset discrimination effects from the masking effects. To assess performance level in these masked conditions, we determined SOA thresholds, that is, we determined the SOA between vernier and grating onset for which subjects reached a score of $75 \%$ of correct responses. To determine these temporal thresholds, we used the adaptative method PEST, previously used for the spatial vernier offset discrimination (see the subsection 'vernier without mask'). The adaptive strategy started with an SOA of $200 \mathrm{~ms}$, which was afterwards either decreased or increased to find each participant's individual threshold. These thresholds were determined within a block of 80 trials. In many conditions, subjective visibility of the preceding vernier was completely abolished. We restricted the PEST procedure to SOAs shorter than $400 \mathrm{~ms}$. If participants were unable to reach a stable threshold value within this series, an offset of $450 \mathrm{~ms}$ was tabulated and used as 'in-discriminability' (for details see Herzog et al, 2001)

When a five-element grating follows the vernier, the later is harder to detect. It becomes almost completely invisible because of the strong masking effect. In this situation, the entire grating can appear for trained participants to be offset in the direction of the vernier (feature inheritance; Herzog and Koch, 2001). In the present case, participants 
were free to make the offset discrimination judgment based on the grating. In general, we did not encourage participants to make judgments using specific cues. Whether subjects based their decision on feature inheritance or on the preceding vernier, performance is significantly worse in both cases compared to shine-through occurring when the vernier was followed by a 25 -element grating (Herzog et al, 2001).

\section{Procedure}

The experiment was conducted in four sessions, a first training session and three experimental sessions in which lorazepam, diazepam, or a placebo were given. During the training session, the experimental paradigm was described. This step ensured that participants understood the task before being under the effects of a drug. The critical vernier duration for each participant was also determined free of all drug effects. Finally, for each participant, the critical SOA was determined, that is, the time between vernier and grating presentation, for a 25 -element grating only (during the training session, the five-element grating was not used). On the following day, during the first experimental session, lorazepam, diazepam, or placebo was given. The remaining two drug conditions were applied 1 and 2 weeks later for the second and third experimental session, respectively. In the three sessions and for each individual, the critical vernier duration was first determined without the use of a mask and then, the two masking conditions were run using this vernier duration.

\section{Participants}

Participants were 12 students from the University of Strasbourg. They had normal or corrected-to-normal visual acuity and were naive as to the aim of the study. They ranged in age from 19 to 28 years (mean age: 22.7 years) and ranged in weight from 50 to $70 \mathrm{~kg}$ (mean weight: $59.5 \mathrm{~kg}$ ). The subjects had no medical illness or history of alcoholism, drug abuse, or tobacco consumption of more than 10 cigarettes per day. They were not chronic users of benzodiazepines and had not taken any medication for at least 15 days. Participants were instructed to abstain from beverages containing caffeine or alcohol during a $24 \mathrm{~h}$ period prior to the study. All participants were tested the morning after an overnight fast. All participants gave written informed consent and were paid for their participation. The protocol was approved by the Faculty Ethics Committee. The study was carried out in accordance with the Declaration of Helsinki.

\section{Drug Treatment}

Each participant received a single dose of placebo, of $0.038 \mathrm{mg} / \mathrm{kg}$ lorazepam, and of $0.3 \mathrm{mg} / \mathrm{kg}$ diazepam with a minimal delay of 8 days between each intake. The order of administration of the placebo, the lorazepam, and the diazepam tablets was counterbalanced across participants. The drug tablets were given orally using a double-blind procedure. The time of administration was defined so that the drug effects peaked at a similar moment $(1 \mathrm{~h}$ after intake for diazepam and $2 \mathrm{~h}$ for lorazepam). In all cases, two tablets were administered, a first one at 07:30 and a second one at 08:30. For the placebo, both tablets were placebos. For lorazepam, the first tablet was lorazepam and the second was a placebo. For diazepam, the first tablet was a placebo and the second was diazepam. Hence, peak action was reached between 09:30 and 11:00 for both drug conditions. Participants were tested during this period.

Stimuli were always presented monocularly to avoid possible oculomotor imbalance induced by either benzodiazepine (Speeg-Schatz et al, 2001).

\section{Sedation Evaluation}

Pupillography. After the visuo-perceptual tasks, the size of the pupil was measured by means of infrared video pupillography. Participants were instructed to look through goggles at an array of infrared light-emitting diodes, which were used to illuminate the pupil. Their heads were maintained immobile with the use of a chin rest and of a forehead support. The test was conducted in a dark room. The pupil image was filmed with an infrared-sensitive videocamera (Canon CI-20 PR) and recorded through a real-time video board CORECO Oculus 300.

The pupil was seen to be bright thanks to the confocal arrangement of the diodes. The detection of the brightest spot, that is, the Purkinje reflex, gave the means to detect the location of the pupil. Several points, corresponding to the left and right edges of the pupil, were then determined and the diameter of the pupil was calculated. The diameter was measured during $11 \mathrm{~min}$ with a sampling frequency of $25 \mathrm{~Hz}$. The camera picture of the participants' eye was displayed on-line on one screen while another screen viewed the changes in function of time of pupil diameter and eye position.

An artifact rejection algorithm was applied to the data in order to remove blinks and high-frequency noise. Afterwards a pupillary unrest index (PUI) was calculated. The PUI corresponds to the pupil's tendency to oscillate. Highfrequency oscillations were excluded from this calculation by averaging the pupil diameter (in $\mathrm{mm}$ ). Hence, variations in the pupil diameter were taken into consideration in the following calculation only if they were below $1.56 \mathrm{~Hz}$. The PUI corresponds to the sum of the absolute differences in pupil diameter averaged across 16 consecutive data points. This sum was then normalized over a $1 \mathrm{~min}$ period (for more details, see Schaeffel et al, 1993; Lüdtke et al, 1998).

Analogue self-rating of sedation (EVA). A set of visual scales was used to assess the participants' subjective state of sedation. Each scale consisted of a $100 \mathrm{~mm}$ ungraduated horizontal line, anchored by contrasting states of mind. Subjects were asked to regard each line as a continuum and to rate their feelings by placing a vertical mark across each line. The scales were scored by measuring the length in millimeters from the positive end of each line to the subject's mark. Nine of these scales assessed complementary aspects of sedation (Bond and Lader, 1974). The mean score of these nine scales was calculated for each individual and was taken as a subjective indication of sedation. 


\section{Statistical Analysis}

In the vernier offset discrimination task, that is, in the absence of masking, analyses of variance were conducted separately on the threshold of vernier offset discrimination and on the duration of vernier presentation. For the masking procedures, analyses of variance were conducted on the SOA intervals, which participants required in order to reach a score of $75 \%$ of correct responses. All analyses were conducted with participants as a random variable and drug (lorazepam, diazepam, placebo) as a within-effect. Grating (five-, 25-element) was an additional within-effect in the masking procedures.

\section{RESULTS}

During the training session, mean vernier duration was $21.7 \mathrm{~ms}$, for a threshold of vernier offset discrimination of $36^{\prime \prime}$. Performance was stable under placebo $(26.7 \mathrm{~ms}$ and $30^{\prime \prime}$, mean of 12 participants, $\mathrm{F}<1$ ). Under diazepam, only one subject was unable to reach a threshold of about $40^{\prime \prime}$, for a vernier duration of less than $100 \mathrm{~ms}$. Performance in the 11 remaining participants was comparable under diazepam $\left(32.7 \mathrm{~ms}\right.$ and $\left.36^{\prime \prime}\right)$ and placebo $\left(27.2 \mathrm{~ms}\right.$ and $32.3^{\prime \prime}$, mean of 11 participants), $\mathrm{F}(1,10)=2.6$, NS for vernier duration, and $\mathrm{F}<1$ for vernier offset discrimination.

Under lorazepam, five subjects were unable to perform vernier offset discrimination with a vernier duration of less

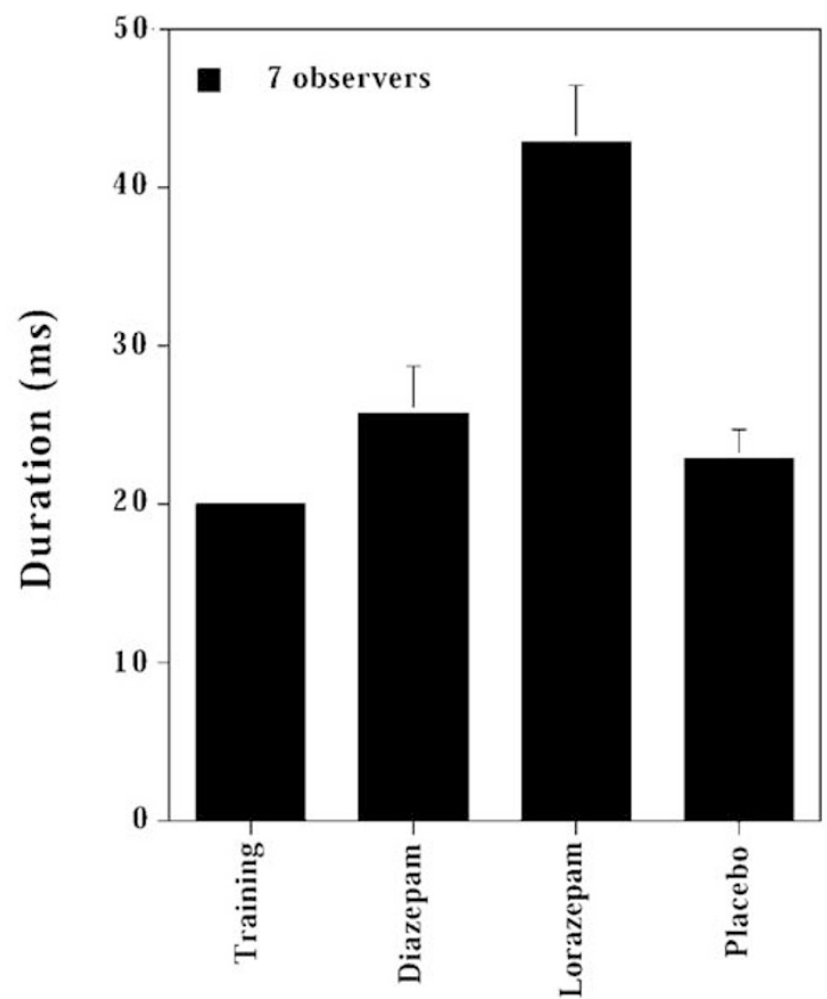

Figure 2 Mean duration for the unmasked vernier in the training, placebo, lorazepam, and diazepam session. One subject under diazepam and five subjects under lorazepam were unable to perform the task with a vernier duration below $100 \mathrm{~ms}$ and hence, were discarded from further investigations. Only the averaged results (with standard errors) of the seven remaining subjects are shown. than $100 \mathrm{~ms}$. Mean vernier duration in the seven remaining participants (Figure 2) was longer under lorazepam $(42.9 \mathrm{~ms})$ than under placebo $(22.9 \mathrm{~ms}), \mathrm{F}(1,6)=21$, $p<0.005$. Mean vernier duration was also significantly larger under lorazepam $(42.9 \mathrm{~ms})$ than under diazepam (25.7 ms), $\mathrm{F}(1,6)=10.8, p<0.05$. Mean offset discrimination was higher in these seven participants when they were under lorazepam $\left(54.1^{\prime \prime}\right)$ than under either placebo $\left(26.4^{\prime \prime}\right)$, $\mathrm{F}(1,6)=4.7, p=0.07$, or diazepam $\left(25.6^{\prime \prime}\right), \mathrm{F}(1,6)=10.4$, $p<0.05$. Hence, for these seven subjects, the main effect of treatment reached significance for both mean vernier duration $(\mathrm{F}(2,12)=11.9, p<0.001)$ and for mean vernier offset discrimination threshold, $\mathrm{F}(2,12)=6.3, p<0.05$.

In the following, we consider only the results of the seven subjects who were successfully able to perform vernier discrimination under lorazepam in the unmasked condition. When the vernier with a fixed offset of $75^{\prime \prime}$ was followed by a 25-element grating, all seven subjects were able to perform the task properly in all three conditions (Figure 3). However, the SOA threshold between vernier and grating onset tended to vary in function of the considered drug, $F(2,12)=2.9, p=0.09$ : the SOA was $43 \mathrm{~ms}$ under placebo and increased slightly but not significantly under diazepam, $69 \mathrm{~ms}, \mathrm{~F}(1,6)=2$, NS. The increase was more important under lorazepam $(118 \mathrm{~ms})$, but still did not reach significance, $\mathrm{F}(1,6)=4.7, p=0.07$. SOAs were also not significantly different for lorazepam and diazepam, $\mathrm{F}(1,6)=1.6$, NS.

Masking effects were stronger with the five-element grating. All seven subjects were able to perform the task when they were under placebo and diazepam. In contrast,

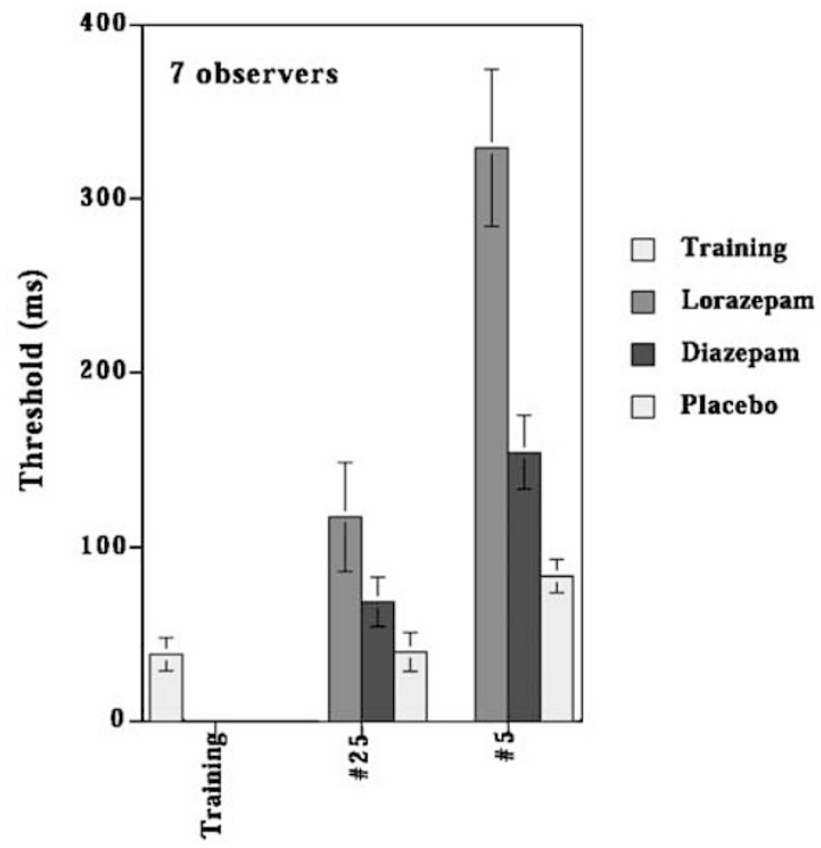

Figure 3 Mean SOA thresholds (with standard errors) as a function of the session (training, placebo, lorazepam, and diazepam), for masks composed of five- or 25-element gratings. Mean results are presented for the seven participants who were able to perform properly in the absence of a mask and under the effects of lorazepam. 
three subjects were unable to reach an SOA threshold of less than $400 \mathrm{~ms}$ under lorazepam and consequently, these participants were attributed the preset SOA value of $450 \mathrm{~ms}$. Under lorazepam and without mask, these three subjects did not differ significantly from the four other subjects regarding either the discrimination threshold offset $\left(62.9^{\prime \prime}\right.$ vs $47.4^{\prime \prime}, \mathrm{F}(1,5)=1.3$, NS) or the vernier duration (42.5 vs $43.3 \mathrm{~ms}, \mathrm{~F}<1$ ).

Under placebo, the SOA thresholds significantly increased by a factor of 1.9 between the 25-element grating ( $43 \mathrm{~ms}$ ) and the five-element grating $(84 \mathrm{~ms}), \mathrm{F}(1,6)=9, p<0.05$. Under diazepam, the SOA increased by a factor of 2.2 between the 25-element grating $(69 \mathrm{~ms})$ and the fiveelement grating $(155 \mathrm{~ms}), \mathrm{F}(1,6)=8.2, p<0.05$. The masking effect of the five-element grating was significantly larger under diazepam than under placebo, $\mathrm{F}(1,6)=14.4, p<0.01$. However, the increase in the masking effect from the 25- to the five-element grating did not differ significantly under placebo and diazepam, that is, the interaction between group and masking condition did not reach significance, $\mathrm{F}(1,6)=2.8$, NS.

Under lorazepam, the SOA increased by a factor of 2.8 between the 25 -element grating $(118 \mathrm{~ms})$ and the fiveelement grating $(330 \mathrm{~ms}), \mathrm{F}(1,6)=14, p<0.01$. The masking effect of the five-element grating was significantly larger under lorazepam $(330 \mathrm{~ms})$ than under either diazepam $(155 \mathrm{~ms}), \quad \mathrm{F}(1,6)=29.9, \quad p<0.005$ or placebo $(84 \mathrm{~ms})$, $\mathrm{F}(1,6)=33.3, p<0.001$. The increase in the masking effect from the 25- to the five-element grating was significantly larger under lorazepam (2.8) than under either diazepam (2.2), $\mathrm{F}(1,6)=13.8, p<0.01$, or placebo $(1.9), \mathrm{F}(1,6)=10$, $p<0.05$. Indeed, the interaction between treatment and masking condition was significant, $\mathrm{F}(2,12)=9.8, p<0.005$.

\section{Sedation Effects}

Under placebo, the mean PUI was 7.9 and the mean EVA was 8.0. These sedation indices increased significantly under both lorazepam $(15.7 ; 38)$ and diazepam $(13 ; 33)$. This resulted in a significant main effect of treatment, $\mathrm{F}(2,22)=6.6, p<0.01$ and $\mathrm{F}(2,22)=24, p<0.001$ for the PUI and the EVA, respectively. Neither the mean PUI nor the mean EVA differed significantly between the two benzodiazepines, $\mathrm{F}(1,11)=3$, NS; $\mathrm{F}(1,11)=1.3$, NS. Finally, the sedation levels were similar under lorazepam and diazepam, whether these levels were measured with or without the masking procedure. No correlations were significant between performance and sedation levels.

\section{DISCUSSION}

The aim of the present study was to examine the effects of two different benzodiazepines on the time course of visual information processing as determined by visual backward masking.

\section{Lorazepam Affects Visual Information Processing of the Unmasked Vernier Target}

In the unmasked condition, five of the 12 participants were unable to reach the predefined performance level when tested under lorazepam. Only one encountered such a difficulty when tested under diazepam, and none if tested under placebo. When considering specifically the seven participants who succeeded in performing the tests under lorazepam, a mean vernier duration of $42.9 \mathrm{~ms}$ was required under lorazepam, whereas vernier durations of only 22.9 and $25.7 \mathrm{~ms}$ were sufficient under placebo and diazepam, respectively (Figure 2). Both visual acuity and accommodation have been reported to be preserved under the effects of lorazepam (Turner, 1973; Speeg-Schatz et al, 2001). In addition, participants treated with similar doses of benzodiazepines have been shown to perform visual tasks without apparent difficulty (Giersch et al, 1997; Giersch \& Lorenceau, 1999; Giersch, 1999, 2001). Consequently, it is very surprising that five out of 12 of our participants were completely unable to perform the task under lorazepam and that the other seven participants performed significantly worse under lorazepam than under diazepam or placebo. This effect cannot solely be attributed to the difficulty of the task and/or a nonspecific effect of the lorazepam drug. Our results show that the performance level was not correlated with sedation level. More importantly, both subjective and objective indicators of sedation levels were similar under lorazepam and diazepam. Despite this, there was a specific and significant deterioration of performance under lorazepam in the processing of visual information, that is, on the discrimination of the vernier offset direction.

One might argue that lorazepam affects attention allocation. However, lorazepam and diazepam have not been shown to differ significantly in tests exploring attentional effects (eg in the cancellation task, Vidailhet et al, 1994, Legrand et al, 1995). Moreover, Boucart et al (2000) compared the effects of lorazepam and diazepam on visual attention with an attentional blink paradigm (RSVP). The doses were identical to those used in the present study and the results showed that lorazepam-treated participants were able to attend to visual information in an efficient way, and even in a more efficient way than under diazepam. Hence, the effects induced by lorazepam in the present study, which differ very markedly from diazepam, can hardly be attributed to a nonspecific attentional deficit.

Vernier offset discrimination relies on the pooling of responses of a large number of neurons, sensitive to spatial position or orientation (eg Westheimer, 1981; Wilson, 1986). Indeed, comparing the position of the two elements of the vernier cannot be achieved with a single neuron. It is possible that lorazepam deteriorates the integration of the two line-segments composing the vernier stimulus. As mentioned, lorazepam enhances the separation of collinear line-segments possibly by reinforcing the line-end signals (Giersch, 1999; Beckers et al, 2001; Giersch, 2001). However, in the present setup, neither the position nor the orientation of the line-ends per se provided sufficient indications on the direction of the vernier offset. On the contrary, the enhancement of line-ends may have prevented the pooling of spatial and/or orientation sensitive neurons and thus, impaired vernier offset discrimination. This hypothesis is consistent with studies suggesting that lorazepam enhances segmentation processes at the cost of integration processes (Giersch and Lorenceau, 1999; Giersch, 1999; Lorenceau et al, submitted). 


\section{Visual Masking Reveals Prolongation of Visual Information Processing under Lorazepam}

The deteriorating effect of lorazepam further increased when verniers were masked with five- or 25-element gratings. In contrast to the placebo condition, which yielded thresholds comparable to the training session, SOA thresholds increased significantly under lorazepam but only moderately under diazepam. This pattern of result was accentuated with the five-element gratings. Compared to placebo, thresholds increased by a factor of 1.6 for 25element gratings if subjects were under diazepam and by a factor of 2.9 if tested under lorazepam. For five-element gratings, thresholds increased by a factor of 1.8 and 3.9 under diazepam and lorazepam, respectively, reaching a mean threshold of $330 \mathrm{~ms}$ under lorazepam. This latter result is most probably an underestimation of performance as three of the participants under lorazepam showed ceiling effects in this condition, that is, SOAs could not be determined and hence, a value of 'only' $450 \mathrm{~ms}$ was attributed. Nevertheless, the surprising result is that masking remained effective even with such long SOA delays. As a reminder, for threshold SOAs, only $75 \%$ of correct responses are reached and consequently, there are still strong masking effects at these SOA intervals. In the literature, masking is described to be effective in high contrast targets for SOAs of no longer than $100 \mathrm{~ms}$. Hence, the fact that in the present study there remains a masking effect at SOAs of $450 \mathrm{~ms}$ in lorazepam-treated participants reveals not only a quantitative but must also be associated to a qualitative difference: in lorazepamtreated individuals, target processing is significantly prolonged and hence, the late coming gratings can still exert their masking effects.

It could be argued that the longer masking effects may be caused by a longer persistence of the target under lorazepam. Longer persistence effects may account also for higher thresholds in the critical flicker-fusion test. Elliott et al (2000) used a $40 \mathrm{~Hz}$ oscillating prime and showed that lorazepam induced a larger priming effect than placebo. Hence, lorazepam-treated participants cannot fuse consecutive frames, as this priming effect requires that alternating frames be separated one from the other. Moreover, the very long SOAs reported in the present study can hardly be accounted for by a retinal persistence effect. Therefore, the prolonging effects shown here under lorazepam may better be explained in terms of a prolonged target processing rather than a longer target persistence.

\section{Mask-Specific Effects}

Under lorazepam, better performance levels were obtained with 25-element gratings compared to five-element gratings. This suggests that the effects of lorazepam are specific to the spatial layout of the gratings and are not caused by some nonspecific masking effect. As already emphasized, the masking energy obtained with a 25-element grating is higher than that obtained with a five-element grating. Hence, the spatial layout rather than the mask energy determines performance level. This conclusion is in contradiction with many studies previously published on masking effects (eg Breitmeyer, 1984; Francis, 2000; Francis and Herzog, in press). In addition to the fact that masking effects are longer under lorazepam, these effects are also significantly longer when the grating is composed of five elements rather than 25. Indeed, when the vernier is followed by a five-element grating, subjects under placebo were able to discriminate appropriately the direction of the offset for SOAs of 40-120 ms. In contrast, only three subjects under lorazepam were able to make such discriminations for SOA intervals shorter than $300 \mathrm{~ms}$, and three subjects were unable to perform the task for SOAs shorter than $400 \mathrm{~ms}$, even though all of these individuals were able to perform correctly in the other conditions (ie with 25-element gratings). Importantly, these three latter subjects did not differ from the other four lorazepamtreated participants when performing the discriminating task in the absence of a mask.

The fact that effects are specific for the respective masks rule out nonspecific factors such as an interruption of decision processing, attentional effects or, as previously mentioned, mask energy. These mask-specific effects are surprising since for untreated observers it has been shown that all types of masks exert similar masking effects if presented for SOAs longer than about $150 \mathrm{~ms}$ (Enns, submitted). Contrary to this, our results show that all lorazepam-treated participants show specific effects even for SOAs $180 \mathrm{~ms}$ and more.

The dramatic deterioration of performance with the fiveelement gratings may be explained by a lorazepam-induced enhancement of the edges of the grating. This hypothesis is consistent with the described effects of lorazepam on the inhibitory connections and may be related to the drug's effects on the processing of contours.

\section{Lorazepam vs Diazepam}

The present results revealed that lorazepam and diazepam yield the same sedation effects as determined subjectively and objectively. These drugs differ, however, both on the performance levels for vernier offset discrimination and on masking effects. Differences between diazepam and lorazepam have previously been described concerning the identification of fragmented pictures (Vidailhet et al, 1994; Legrand et al, 1995; Wagemans et al, 1998), priming effects (Sellal et al, 1992; Vidailhet et al, 1994; Legrand et al, 1995), and detection of line discontinuities (Beckers et al, 2001). The link between the present findings and the effects of lorazepam on contour processing is supported by studies reporting an absence of effect of diazepam on the detection of line discontinuities (Beckers et al, 2001). Why both drugs exert such different effects is still not clear. It is not simply that lorazepam is more potent than diazepam as both drugs have similar negative effects on explicit memory (Sellal et al, 1992; Vidailhet et al, 1994; Legrand et al, 1995). Furthermore, diazepam has been shown to be more deleterious than lorazepam at certain occasions (Boucart et al, 2000). Both drugs may in fact affect visual information processing in a qualitatively different manner, possibly by the possession of different affinities for a variety of subtypes of $\mathrm{GABA}_{\mathrm{A}}$ receptors (Mohler et al, 2002). The origin of these differences has yet to be clarified. 


\section{CONCLUSIONS}

The lorazepam drug has significant and deleterious effects on visual information processing. The dramatic prolongation of the processing mechanisms does not result from nonspecific factors, as our findings are specific to a given type of mask grating. We have shown that under lorazepam, 25-element gratings (higher energy) yield less masking effects than five-element gratings (lower energy). Consequently, lorazepam probably influences spatial information related to either contour-processing, grouping, or figureground segmentation. In the present study, the difference in performance levels could not be explained by a nonspecific effect of sedation induced by lorazepam, as sedation indices were comparable with both drugs. The combination of backward masking procedures and the intake of lorazepam has here been shown to be a promising technique for the investigation of how the molecular mechanisms of the inhibitory system, via $\mathrm{GABA}_{\mathrm{A}}$ receptors, affect the timing of visual information processing.

\section{ACKNOWLEDGEMENTS}

MHH's research was financed by the SFB 517 'Neurocognition' of the Deutsche Forschungsgemeinschaft (DFG). AG's research was financed by INSERM and by the University Hospital of Strasbourg. We thank Marc Repnow for his invaluable help with technical support, Daniela Hoegl for help in data management, and Dr Marie Welsch for the medical examination of the healthy volunteers.

\section{REFERENCES}

Bachmann T (1994). Psychophysiology of Visual Masking. Nova Science Publishers: New York.

Beckers T, Wagemans J, Boucart M, Giersch A (2001). Different effects of lorazepam and diazepam on perceptual integration. Vision Res 41: 2297-2303.

Berlin I, Warot D, Hergueta T, Molinier P, Bagot C, Puech AJ (1993). Comparison of the effects of zolpidem and triazolam on memory functions, psychomotor performances, and postural sway in healthy subjects. J Clin Psychopharmacol 13: 100-106.

Bond A, Lader M (1974). The use of analogue scales in rating subjective feelings. Br J Med Psychol 47: 211-218.

Boucart M, de Visme P, Wagemans J (2000). Effect of benzodiazepine on temporal integration in object perception. Psychopharmacology 152: 249-255.

Breitmeyer BG (1984). Visual Masking. Oxford University Press: New York.

Breitmeyer BG, Ogmen H (2000). Recent models and findings in backward visual masking: a comparison, review, and update. Percept Psychophys 62: 1572-1595.

Curran HV, Gardiner JM, Java RI, Allen D (1993). Effects of lorazepam upon recollective experience in recognition memory. Psychopharmacology 110: 374-378.

Curran HV, Pooviboonsuk P, Dalton JA, Lader MH (1998). Differentiating the effects of centrally acting drugs on arousal and memory: an event-related potential study of scopolamine, lorazepam and diphenhydramine. Psychopharmacology 135: 27-36.

Danjou P, Warot D, Hergueta T, Lacomblez L, Bouhours P, Puech AJ (1992). Comparative study of the psychomotor and antistress effects of ritanserin, alprazolam and diazepam in healthy subjects: some trait anxiety-independent responses. Int Clin Psychopharmacol 7: 73-79.
Elliott MA, Becker C, Boucart M, Muller HJ (2000). Enhanced GABA(A) inhibition enhances synchrony coding in human perception. Neuroreport 11: 3403-3407.

Emre M, Groner M, Hofer D, Groner R, Fisch HU (1989). Effects of benzodiazepines on forward and backward visual masking. Clin Vision Sci 4: 257-263.

Enns JT. Object substitution and its relation to other forms of visual masking. Vision Res (submitted).

Enns JT, Di Lollo V (2000). What's new in visual masking? TICS 4: 345-352.

Fisch HU, Groner M, Groner R, Menz C (1983). Influence of diazepam and methylphenidate on identification of rapidly presented letter strings: diazepam enhances visual masking. Psychopharmacology 80: 61-66.

Francis G (2000). Quantitative theories of metacontrast masking. Psychol Rev 107: 1-18.

Francis G, Herzog MH. Testing quantitative models of backward masking. Psychonomic Bull Rev (in press).

Giersch A (1999). A new pharmacological tool to investigate integration processes. Visual Cogn 6: 267-297.

Giersch A (2001). The effects of lorazepam on visual integration processes: how useful for neuroscientists? Visual Cogn 8: 549-563.

Giersch A, Boucart M, Danion JM (1997). Lorazepam, a benzodiazepine, induces atypical distractor effects with compound stimuli: A role for line-ends in the processing of compound letters. Visual Cogn 4: 337-372.

Giersch A, Lorenceau J (1999). Effects of a benzodiazepine, lorazepam, on motion integration and segmentation: an effect on the processing of line-ends? Vision Res 39: 2017-2025.

Herzog MH, Ernst UA, Etzold A, Eurich CW (2003). Local interactions in neural networks explain global effects in the masking of visual stimuli. Neural Comput 15: 2091-2113.

Herzog MH, Fahle M, Koch C (2001). Spatial aspects of object formation revealed by a new illusion, shine-through. Vision Res 41: $2325-2335$.

Herzog MH, Koch C (2001). Seeing properties of an invisible object: feature inheritance and shine-through. Proc Natl Acad Sci USA 98: 4271-4275.

Legrand F, Vidailhet P, Danion JM, Grangé D, Giersch A, Van Der Linden $\mathrm{M}$ et al (1995). Time course of the effects of diazepam and lorazepam on perceptual priming and explicit memory. Psychopharmacology 118: 475-479.

Lorenceau J, Giersch A, Series P. Dynamic competition between contour integration and contour segmentation probed with moving stimuli. Vision Res (submitted).

Lüdtke H, Wilhelm M, Adler F, Schaeffel H, Wilhelm H (1998). Mathematical procedures in data recording and processing of pupillary fatigue waves. Vision Res 38: 2889-2996.

MacKay DM (1973). Lateral interaction between neural channels sensitive to texture. Nature 245: 159-161.

Macknik SL, Martinez-Conde S, Haglund MM (2000). The role of spatiotemporal edges in visibility and visual masking. Proc Natl Acad Sci USA 97: 7556-7560.

MacNab MW, Foltz EL, Sweitzer J (1985). Evaluation of signal detection theory on the effects of psychotropic drugs on critical flicker-fusion frequency in normal subjects. Psychopharmacology 85: 431-435.

Maddock RJ, Casson EJ, Lott LA, Carter CS, Johnson CA (1993). Benzodiazepine effects on flicker sensitivity: role of stimulus frequency and size. Prog Neuropsychopharmacol Biol Psychiatry 17: 955-970.

McCarter A, Roehrs T (1976). A spatial frequency analogue to mach bands. Vision Res 16: 1317-1321.

Mohler H, Fritschy JM, Rudolph U (2002). A new benzodiazepine pharmacology. J Pharmacol Exp Ther 300: 2-8. 
Sagi D, Hochstein S (1985). Lateral inhibition between spatially adjacent spatial-frequency channels? Percept Psychophys 37: 315-322.

Schaeffel F, Wilhelm H, Zrenner E (1993). Inter-individual variability in the dynamics of natural accommodation in humans: relation to age and refractive errors. J Physiol 461: 301-320.

Sellal F, Danion JM, Kauffmann-Muller F, Grange D, Imbs JL, Van der Linden $M$ et al (1992). Differential effects of diazepam and lorazepam on repetition priming in healthy volunteers. Psychopharmacology 108: 371-379.

Speeg-Schatz C, Giersch A, Boucart M, Gottenkiene S, Tondre M, Kauffmann-Muller F et al (2001). Visual effects of lorazepam on the oculomotor balance. Binocular Vision Strabismus Quart 16: 99-104.

Taylor MM, Creelman CD (1967). PEST: efficient estimates on probability functions. J Acoust Soc Am 41: 782-787.

Turner P (1973). Clinical pharmacological studies on lorazepam. Curr Med Res Opin 1: 262.

Vidailhet P, Danion JM, Kauffman-Muller F, Grangé D, Giersch A, Van Der Linden M et al (1994). Lorazepam and diazepam effects on memory acquisition in priming tasks. Psychopharmacology 115: 397-406.

Wagemans J, Notebaert W, Boucart M (1998). Lorazepam but not diazepam impairs identification of pictures on the basis of specific contour fragments. Psychopharmacology 138: 326-333.

Westheimer G (1981). Progress in Sensory Physiology 1. Visual Hyperacuity. Springer Verlag: Berlin.

Wilson HR (1986). Responses of spatial mechanisms can explain hyperacuity. Vision Res 26: 453-469. 